ORIGINAL ARTICLE

\title{
Prevalence and relevance of EBV latency in nasopharyngeal carcinoma in Israel
}

\author{
G Bar-Sela, A Kuten, I Minkov, E Gov-Ari, O Ben-Izhak
}

J Clin Pathol 2004;57:290-293. doi: 10.1136/icp.2003.013094

See end of article for authors' affiliations

Correspondence to:

Dr G Bar-Sela, Department of Oncology, Rambam Medical Centre, PO Box 9602, Haifa 31096 Israel: g_barsela@rambam. health.gov.il

Accepted for publication 23 October 2003
Background: Nasopharyngeal carcinoma (NPC) is frequently associated with Epstein-Barr virus (EBV). The incidence of NPC in Western countries is lower than in the Far East, and EBV latency in NPC is less prevalent. Israel, as a part of the Mediterranean area, is one of the countries with an intermediate risk for NPC.

Methods: Immunohistochemistry (IHC) for latent membrane protein 1 (LMP-1) and in situ hybridisation (ISH) for EBV encoded RNA (EBER) were used to evaluate the prevalence and possible prognostic value of EBV latency among Israeli patients with NPC. Forty five patients with different NPC histologies were studied.

Results: LMP-1 IHC was positive in six samples only, all with undifferentiated histology. EBER ISH was positive in 40 of the 45 samples. According to histological type, three of five patients with squamous cell carcinoma were EBV positive and 37 of 40 non-keratinising and undifferentiated carcinoma cases were positive. Although EBV was more prevalent in patients with non-squamous carcinoma, the difference was not significant, probably because of the small number of patients with keratinising carcinoma. With regard to the clinical categories and survival, no significant difference could be detected between patients who were positive or negative for EBER ISH. No association was found between EBV latency and patient sex, age, origin, stage, or survival.

Conclusions: NPC in Israel is highly associated with EBV latency as detected by EBER ISH. LMP-1 IHC is considerably less sensitive in detecting EBV latency in NPC among the same patient group.
$\mathrm{T}$ he Epstein-Barr virus (EBV) was discovered 38 years ago by electron microscopy of cells cultured from Burkitt's lymphoma tissue by Epstein, Achong, and Barr. ${ }^{1}$ Six years later, in 1970, EBV DNA was detected in tissues from patients with nasopharyngeal carcinoma (NPC). ${ }^{2}$ NPC is an aggressive tumour that originates within the postnasal space. It has been classified by the World Health Organisation (WHO) into three types: squamous cell carcinoma (type 1), non-keratinising carcinoma (type 2), and undifferentiated carcinoma (type 3). Tumours of types 2 and 3 are clearly associated with EBV, whereas virus involvement in NPC type 1 is more controversial. ${ }^{3}$ The staging of NPC according to the American Joint Committee of Cancer (AJCC) ${ }^{4}$ is well known to have important prognostic value. ${ }^{5}$ The tumour has a multifactorial aetiology, including virological, environmental, and genetic components. Incidence varies greatly with geographical location, age, and race. NPC occurs mainly in Southeast Asia and is one of the predominant tumours in the population as a whole in Southern China. In these areas, the incidence may reach 80 cases/100 000 population each year. ${ }^{6}$ Intermediate incidence is noted in Israel, along with other countries in the Mediterranean basin (North Africa, southern Italy, Greece, Turkey). ${ }^{5}$ Most of the patients diagnosed with NPC in Israel have WHO type 2 or 3 histology and advanced stage. $^{78}$

Only two viral proteins are known to be expressed in NPC, the EBV nuclear antigen 1 and the latent membrane protein 1 (LMP-1). ${ }^{9}$ LMP-1 of EBV is thought to be a viral oncogene on the basis of its transforming capability; it is associated with cellular phenotypic modifications of EBV infected B cells, inducing the expression of activation markers and adhesion molecules. ${ }^{10}$ In situ hybridisation (ISH) techniques that target viral DNA or mRNA are of limited use in NPC because low genomic copy numbers and low mRNA transcription in latent infections make the procedure technically demanding. Conversely, the abundant expression of nuclear EBV encoded
RNAs (EBERs) in latently infected cells makes them ideal targets for ISH. The use of digoxigenin labelled antisense EBERl transcript probes in formalin fixed, paraffin wax embedded specimens has demonstrated abundant expression of EBERl in primary NPC and metastatic NPC. ${ }^{11}$

\section{"The abundant expression of nuclear Epstein-Barr virus encoded RNAs in latently infected cells makes them ideal targets for in situ hybridisation"}

Our study was performed to assess EBV status in Israeli patients with NPC, using LMP-1 immunohistochemistry (IHC) and EBER ISH. We also attempted to correlate the results with the clinical data.

\section{PATIENTS AND METHODS \\ Patients}

We obtained paraffin wax embedded blocks of NPC from 45 patients treated in the department of oncology at Rambam Medical Centre in Haifa, Israel, between 1971 and 1997, from the archives of the department of pathology in the same medical centre. Follow up records for a minimum period of four years after completion of treatment or until diagnosis of recurrence were required. All tissue sections were available for LMP-1 IHC and for EBER ISH. The latest disease status was known for 43 patients only (follow up on two patients was not found). Treatment consisted of irradiation only or combined irradiation and chemotherapy. The patients'

Abbreviations: AJCC, American Joint Committee of Cancer; EBER, nuclear Epstein-Barr virus encoded RNA; EBV, Epstein-Barr virus; IHC, immunohistochemistry; ISH, in situ hybridisation; LMP-1, latent membrane protein 1; NPC, nasopharyngeal carcinoma; WHO, World Health Organisation 
hospital charts and the national tumour registry records were reviewed to determine clinical stage, distant and nodal metastatic status, and survival. Clinical stage was determined according to the AJCC criteria, ${ }^{4}$ but complete clinical stage was found for only 33 patients. Data regarding recurrences were missing in some charts and, therefore, were not included in the statistical analysis. Statistical analysis of survival was performed for alive without disease (all with follow up of more than five years), dead of disease, and dead without disease.

\section{Immunohistochemistry}

The tissue sections were dewaxed in xylene twice for 10 minutes each, then rehydrated in graded alcohols and rinsed in tap water. Before immunostaining for LMP-1, slides were microwaved at $92^{\circ} \mathrm{C}$ for 15 minutes in EDTA buffer $(\mathrm{pH}=8)$ (Zymed Laboratory, San Francisco, California, USA), followed by cooling for 20 minutes at room temperature. The samples were stained in the Ventana immunostainer (NexES), according to the manufacturer's instructions. A monoclonal antibody to LMP-1 (Dako, Glostrup, Denmark) was used at a dilution of $1 / 20$. A case of Burkitt's lymphoma was used as a positive control for LMP-1 and the negative control comprised substituting the primary antibody with normal rabbit serum.

\section{ISH for latent EBV infection}

ISH was performed using the EBV probe ISH kit (Novocastra Labs, Newcastle upon Tyne, UK) according to the manufacturer's instructions. Briefly, after the sections were dewaxed, dehydrated, and predigested with proteinase K, hybridisation solution containing the fluorescein conjugated EBV (EBER) nucleic acid probe was applied. After application of alkaline phosphatase conjugated antibody to fluorescein isothiocyanate, BCIP/NBT (bromochloroindolylphosphate/nitroblue tetrazolium chloride) was applied as the chromogen. Slides were then lightly counterstained with haematoxylin. Dark brown nuclear staining identified a positive hybridisation signal. Each specimen was scored for EBER as 0 (negative), 1 $(<10 \%$ of tumour nuclei EBER positive), 2 (10-50\% positive), or 3 ( $>50 \%$ positive). Positive control slides were supplied by the manufacturer (Hodgkin disease). A negative control was run for each specimen by replacing the EBV probe with a negative control probe.

\section{Statistical analysis}

Data were analysed using SPSS 11.0 software. The $\chi^{2}$ test was used to compare categorical variables between two groups and the $t$ test to compare means of continuous variables. Kaplan-Meier survival analysis was performed to compare EBER positive and negative patients.

\section{RESULTS}

The median age at diagnosis of the 45 patients ( 34 males, 11 females) studied was 45 years (mean, 42; range, 10-76). All the patients without evidence of disease at the end of treatment had more than four years of follow up. The maximum period of follow up was 21 years, whereas the median follow up was five years for the entire group and 10 years for the patients who were still alive. Table 1 shows the demographic details and tumour characteristics. Most patients (23 of 37 ) with known staging were in stage IV. The most common histology was undifferentiated carcinoma (30 of 45). Median survival time for the entire group was 11.5 years.

Table 2 shows the results of the EBV studies. LMP-1 IHC was positive in six samples only and all had type 3 histology (fig 1).
Table 1 Patient demographics and tumour characteristics

\begin{tabular}{ll}
\hline Characteristic & No. of patients (\%) \\
\hline Sex & $34(75)$ \\
Male & $11(25)$ \\
Female & \\
Ethnic origin & $35(77)$ \\
Jewish & $10(23)$ \\
Arabic & $9(20)$ \\
Age at diagnosis & $29(64)$ \\
$\leq 21$ & $7(16)$ \\
$22-59$ & $5(11)$ \\
$\geqslant 60$ & $10(22)$ \\
WHO type & $30(67)$ \\
1 (squamous) & $1(2)$ \\
2 (non-keratinising) & $4(9)$ \\
3 (undifferentiated) & $8(18)$ \\
Stage & $9(20)$ \\
1 & $5(11)$ \\
2 & $6(13)$ \\
3 & $12(27)$ \\
$4 a$ & \\
$4 b$ & Co \\
Unknown & \\
\hline WHO, World Health Organisation.
\end{tabular}

Table 2 Results of Epstein-Barr virus (EBV) studies

\begin{tabular}{ll}
\hline EBV status & No. of patients (\%) \\
\hline $\begin{array}{l}\text { LMP-1 } \\
\text { Negative } \\
\text { Positive }\end{array}$ & $39(87)$ \\
EBER* $^{*}$ & $6(13)$ \\
0 & \\
$1(1-10 \%)$ & $5(11)$ \\
$2(10-50 \%)$ & 0 \\
$3(\geqslant 50 \%)$ & $2(4)$ \\
\end{tabular}

*Percentage of positively stained cells in each specimen.

EBER, nuclear EBV encoded RNA; LMP-1, latent membrane protein 1

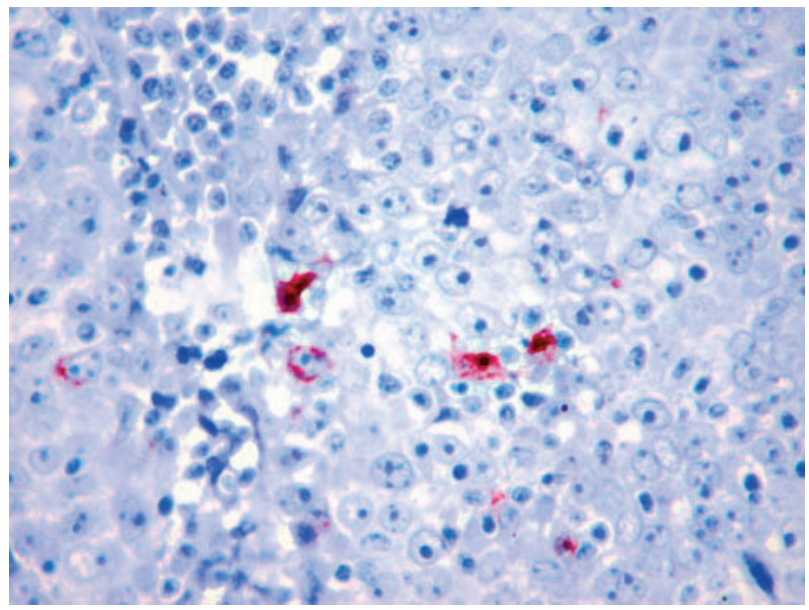

Figure 1 Cytoplasmic staining of Epstein-Barr virus latent membrane protein 1 by immunohistochemistry in several cells; original magnification, $\times 350$.

EBER ISH was positive in 40 of the 45 samples (fig 2). According to histological type, three of five patients with squamous cell carcinoma were EBV positive and 37 of 40 with non-keratinising and undifferentiated carcinoma were EBV positive. Although there was a tendency for a greater proportion of patients with non-squamous carcinoma to be 

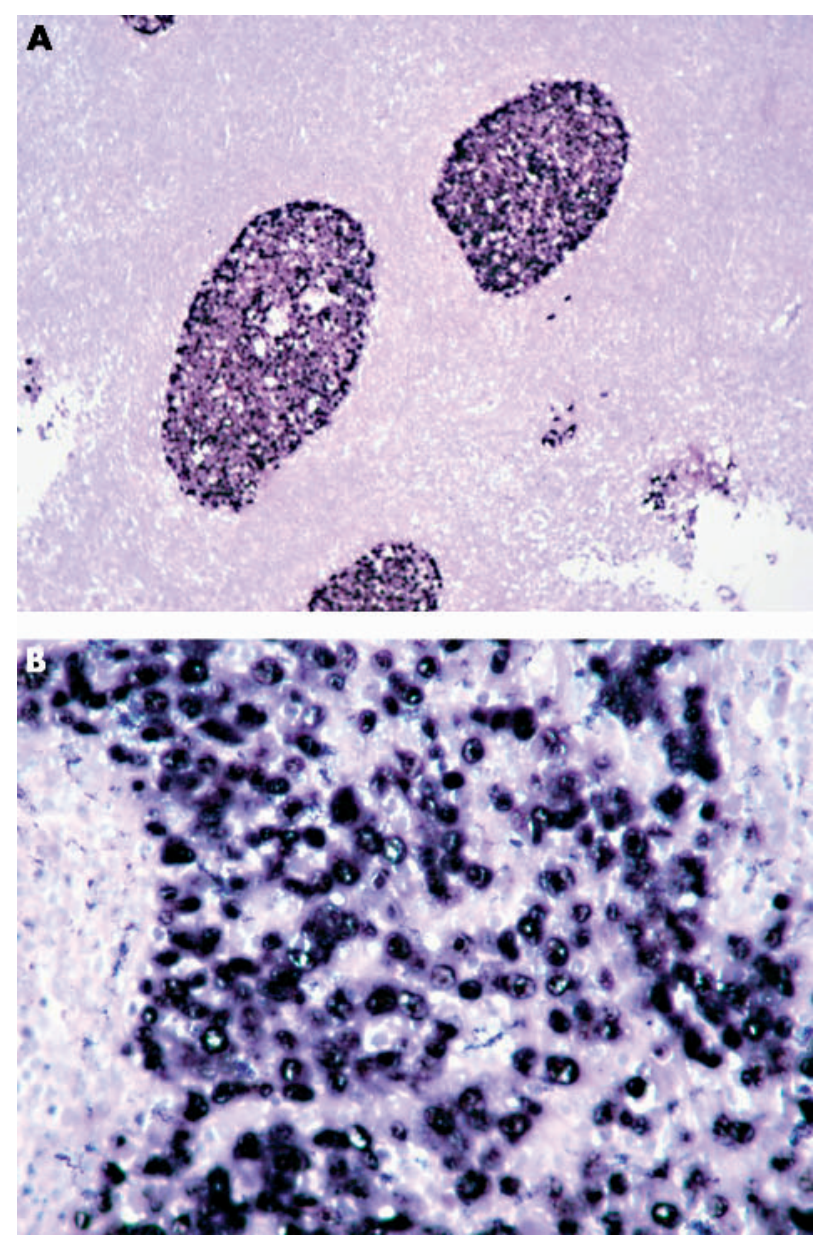

Figure 2 (A) Nests of undifferentiated nasopharyngeal carcinoma in lymphoplasmocytic stroma. Tumour cells are strongly stained by in situ hybridisation to detect nuclear Epstein-Barr virus encoded RNA (EBER); original magnification, $\times 80$. (B) High magnification of nuclear staining for EBER by in situ hybridisation; original magnification, $\times 350$ ).

EBV positive, the results were not significant (squamous carcinoma (type 1) $v$ non-squamous (types 2 and 3), $\mathrm{p}=0.087$ ), probably because of the small number of patients with squamous histology. Among those patients who were EBV positive, almost all (38 of 40 ) had $>50 \%$ tumour nuclei that were EBER positive. Of the two patients with $<50 \%$ of tumour nuclei positive by EBER ISH, one had type 1 histology and one type 2 histology. No significant differences were detected between patients who were positive and negative for EBER ISH with regard to clinical category and survival.

\section{DISCUSSION}

Our study confirms the general association of EBV with NPC and demonstrates that EBER in situ hybridisation detects the presence of latent EBV in tumour tissue in most cases. LMP-1 immunohistochemical expression depends on the concentration of the LMP-1 protein in the cytoplasm of the tumour cells. In contrast, EBER ISH relies on the natural biological amplification of the target sequence, resulting in numerous EBER transcripts. Our results show that EBER ISH is much more sensitive for detecting latent EBV infection in NPC samples. Although one of the three positive EBER squamous carcinomas in our study showed staining of less than $50 \%$ of cells, this finding is not sufficient to conclude that squamous

\section{Take home messages}

- In Israel, nasopharyngeal carcinoma (NPC) is highly associated with Epstein-Barr virus (EBV) latent infection, as it is Southeast Asia

- EBV encoded RNA in situ hybridisation detected EBV in 40 of the 45 patients studied, whereas the detection of latent membrane protein 1 by immunohistochemistry was much less sensitive, detecting EBV in only six of the 45 NPC samples

carcinomas have a lower percentage of positive EBER cells than non-squamous NPCs, as suggested by other studies. ${ }^{12}$

There were five cases of NPC where EBV was not detected by EBER ISH. Two of these cases had WHO type 1 histology and the other three had WHO type 3 histology. EBER ISH is more sensitive than the polymerase chain reaction for detecting EBV latency in tissues, ${ }^{13}$ and enables the localisation of the EBV signal in the tumour cells.

\begin{abstract}
"Although there has been serological evidence of EpsteinBarr virus (EBV) antibodies in patients with nasopharyngeal carcinoma (NPC) in Israel, no previous study has detected EBV in tumour tissue in Israeli patients with NPC"
\end{abstract}

The association between EBV and NPC has been well documented. Most studies are from Southeast Asia, ${ }^{12}{ }^{14}$ but two small recent studies from the Mediterranean basin found an association between EBV latency and NPC. The Spanish trial studied 30 specimens from patients with NPC, 29 of which were positive by EBER ISH, but only 13 were positive by LMP-1 IHC. ${ }^{15}$ The Italian study found that 16 of 24 samples of non-keratinising (types 2 and 3) NPC were positive by EBER ISH. ${ }^{16}$ The results of our present study in an Israeli population, in which 40 of 45 patients were positive by EBER ISH, are similar to these last two studies, although only six of our 45 patients were positive for LMP-1 IHC. This low incidence of LMP-1 in NPC has also been reported by others. ${ }^{17}$ Although there has been serological evidence of EBV antibodies in patients with NPC in Israel, ${ }^{18}{ }^{19}$ no previous study has detected EBV in tumour tissue in Israeli patients with NPC.

The group of five patients negative for EBV is probably too small to determine prognostic differences between the two groups. Other studies with larger numbers of patients negative for EBV found no prognostic value for EBV status. ${ }^{5}$ The only prognostic association between EBV status and survival is the association with different histology. The influence of histological type on outcome is not clear. In general, Chinese studies have found no differences between WHO type 1, 2, and 3 histologies, but cases of WHO type 1 histology are rare $(<5 \%) .{ }^{17}$ In series where both types are well discriminated, patients with WHO type 1 histology do worse in both local control and overall survival. ${ }^{20}$ In our current study, only five of the 45 (11\%) patients had keratinising NPC. In a previous Israeli study of patients with $\mathrm{NPC}^{7} 21 \%$ of patients had keratinising histology. In a review article, ${ }^{17}$ type 1 histology was less than $1 \%$ in the Far East and close to $25 \%$ in the West.

Clonal EBV genomes are found in the early preinvasive dysplastic lesions or carcinoma in situ, indicating that EBV infection precedes the development of malignant invasive tumours. ${ }^{21}$ Our results indicate that all types of NPC among the Israeli population are variants of an EBV associated malignancy. This viral latency is a crucial phenomenon in the development of NPC, in Israel, as in other parts of the world. 


\section{CONCLUSION}

NPC in Israel is highly associated with EBV latency as detected by EBER ISH. LMP-1 IHC is considerably less sensitive in detecting EBV latency in NPC among the same patient group.

\section{ACKNOWLEDGMENT}

The authors thank Mrs M Perlmutter for her help in the preparation of this paper.

\section{Authors' affiliations}

G Bar-Sela, A Kuten, Department of Oncology, Rambam Medical Centre and Faculty of Medicine, Technion-Israel Institute of Technology, Haifa 31096, Israel

I Minkov, O Ben-Izhak, Department of Pathology, Rambam Medical Centre and Faculty of Medicine

E Gov-Ari, Department of Otolaryngology, Rambam Medical Centre and Faculty of Medicine

\section{REFERENCES}

1 Epstein MA, Achong BC, Barr YM. Virus particles in cultured lymphoblasts from Burkitt's lymphoma. Lancet 1964;1:702-3.

2 Zur Hausen H, Schulte-Holthausen H, Klein G, et al. EBV DNA in biopsies of Burkitt tumours and anaplastic carcinomas of the nasopharynx. Nature 1970;228:1056-8.

3 Pathmanathan R, Prasad U, Chandrika G, et al. Undifferentiated, nonkeratinizing and squamous cell carcinoma of the nasopharynx. Variants of Epstein-Barr virus-infected neoplasia. Am J Pathol 1995; 146:1355-67.

4 AJCC Cancer staging handbook. In: AJCC cancer staging manual, 6th ed. New York: Springer, 2002:47-61.

5 Fandi A, Altun M, Azli N, et al. E.Nasopharyngeal cancer: epidemiology, staging, and treatment Semin Oncol 1994;21:382-97.

6 Le Roux F, Joab I. Epstein-Barr virus and nasopharyngeal carcinoma. EpsteinBarr Virus Report 1998;5:53-7.
7 Rahima M, Rakowsky E, Barzilay J, et al. Carcinoma of the nasopharynx: an analysis of 91 cases and comparison of differing treatment approaches. Cancer 1986;58:843-9.

8 Kuten A, Cohen Y, Lavie R, et al. Update on nasopharyngeal carcinoma in northern Israel. In: Strahlentherapie und onkologie, 10th ed. Munich: Urban \& Vogel, 1994:565-70.

9 Young LS, Dawson CW, Clark D, et al. Epstein-Barr virus gene expression in nasopharyngeal carcinoma. J Gen Virol 1988;69:1051-65.

10 Rickinson A, Kieff E. Epstein Barr virus. In: Fields N, Knipe D, eds. Virology. Philadelphia PA: Lippincott-Raven Press Ltd, 1996:2397-446.

11 Wu T-C, Mann RB, Epstein Jl, et al. Abundant expression of EBER1 small nuclear RNA in nasopharyngeal carcinoma. Am J Pathol 1991;138:1461-9.

12 Tsai S-T, Jin Y-T, Su I-J. Expression of EBER1 in primary and metastatic nasopharyngeal carcinoma tissues using in situ hybridization: a correlation with WHO histologic subtypes. Cancer 1996;77:231-6.

13 Tsai S-T, Jin Y-T, Mann RB, et al. Epstein-Barr virus detection in nasopharyngeal tissues of patients with suspected nasopharyngeal carcinoma. Cancer 1998;82:1449-53.

14 Zong YS, Sham JS, Ng MH, et al. Immunoglobulin A against viral capsid antigen of Epstein-Barr virus and indirect mirror examination of the nasopharynx in the detection of asymptomatic nasopharyngeal carcinoma. Cancer 1992;69:3-7.

15 Plaza G, Manzanal Al, Fogue L, et al. Association of Epstein-Barr virus and nasopharyngeal carcinoma in Caucasian patients. Ann Otol Rhinol Laryngol 2002;111:210-16

16 Gabusi E, Lattes C, Fiorentino M, et al. Expression of Epstein-Barr virusencoded RNA and biological markers in Italian nasopharyngeal carcinomas. J Exp Clin Cancer Res 2001;20:371-6.

17 Nicholls JM. Nasopharyngeal carcinoma: classification and histologic appearances. Adv Anat Pathol 1997;4:71-84.

18 Nageris B, Elidan J, Hansen MC, et al. Nasopharyngeal carcinoma among the population in Jerusalem. Am J Otolaryngol 1994;15:190-2.

19 Bogger-Goren S, Gotleib-Stematsky T, Rachmina M, et al. Nasopharyngeal carcinoma in Israel: epidemiology and Epstein-Barr virus-related serology. Eur J Cancer Clin Oncol 1987;23:1277-81.

20 Olmi P, Cellai E, Chiavacci A, et al. Computed tomography in nasopharyngeal carcinoma. Part I: T-stage conversion with CT staging. Part II. Impact on survival. Int J Radiat Oncol Biol Phys 1990;19:1171-82.

21 Pathmanathan R, Prasad U, Sadler R, et al. Clonal proliferations of cells infected with Epstein-Barr virus in preinvasive lesions related to nasopharyngeal carcinoma. N Engl J Med 1995;333:693-8. 